\title{
Fatores Genéticos e de Ambiente Sobre o Intervalo de Partos de Cabras Leiteiras no Semi-Árido Nordestino
}

\author{
José Lindenberg Rocha Sarmento1, Edgard Cavalcanti Pimenta Filho², Maria Norma Ribeiro3, \\ Cláudio Vieira Araújo ${ }^{4}$, Fernanda Cristina Breda ${ }^{5}$, Aldrin Vieira Pires ${ }^{6}$, Rodolpho de Almeida \\ Torres Filho ${ }^{5}$, Robledo de Almeida Torres $^{7}$
}

\begin{abstract}
RESUMO - Objetivou-se estudar a influência de efeitos genéticos e ambientais sobre o intervalo de partos, utilizando-se 486 registros de parição de cabras leiteiras mestiças criadas entre 1988 e 1996, no Estado da Paraíba. Os efeitos ambientais foram analisados pelo procedimento GLM do SAS por meio de um modelo que incluiu os efeitos fixos de grupo contemporâneo e tipo de parto, as covariáveis idade da cabra ao parto e duração da lactação e os efeitos aleatórios de bode e cabra dentro de bode e erro. O programa MTDFREML foi usado para estimar os parâmetros genéticos sob modelo animal, que incluía os mesmos efeitos fixos e covariáveis do modelo anterior. Como aleatórios foram considerados os efeitos genético aditivo, permanente de meio e erro. O intervalo de parto médio obtido foi 275,55 $\pm 60,96$ dias. Os efeitos de grupo contemporâneo e duração da lactação influenciaram o intervalo de partos. As estimativas de herdabilidade e repetibilidade foram de baixa magnitude, 0,09 e 0,09, respectivamente, indicando que reduções mais rápidas nessa característica seriam obtidas por meio de melhorias no manejo.
\end{abstract}

Palavras-chave: caprinos, característica reprodutiva, herdabilidade, repetibilidade

\section{Genetic and Environmental Factors Affecting the Kidding Interval of Dairy Goats in the Semi-Arid Northeastern}

\begin{abstract}
The aims of this study were evaluate influence of the environmental and genetic effects on the kidding interval, using 486 records of kidding crossbred dairy goats obtained from 1988 to 1996 in Paraiba State. The environmental effects were analyzed by means of the procedure GLM of the SAS with a model that included as fixed factors the contemporary group and kidding type, and covariates age of goat at kidding and lactation length. Buck, goat inside of the buck and error were included as random effects. MTDFREML program was used in the analysis with an animal model containing the contemporary group and kidding type, the linear and quadratic effects of age of kidding and lactation length as fixed effects. Animal and permanent environmental and error were included as random effects. The least-square means of kidding interval was $275.55 \pm 60.96$ days. Kidding interval was affected by the contemporary group and lactation length effects. Estimates of heritability and repeatability were low, 0.09 and 0.09 , respectively, showing that smaller kidding intervals would be obtained by better management.
\end{abstract}

Key Words: goats, heritability, reproductive trait, repeatability

\section{Introdução}

Apesar de destacar-se como a maior produtora de caprinos do país, a Região Nordeste apresenta índices zootécnicos ainda baixos. Principalmente em razão da não utilização de tecnologias compatíveis com a região. Dessa forma, o conhecimento dos elementos que interferem direto ou indiretamente nestes índices podem auxiliar na obtenção de maiores eficiências produtiva e reprodutiva dos rebanhos.
Muitos pesquisadores vêm buscando técnicas adequadas de exploração e manejo para a caprinocultura, que, juntamente com linhas de créditos compatíveis com a realidade da maioria dos produtores e um mercado potencial emergente para produtos e derivados da espécie, poderiam mudar o destino da economia regional.

O intervalo de partos está relacionado à viabilidade econômica de uma exploração, bem como ao ganho genético anual, uma vez que o aumento nessa carac-

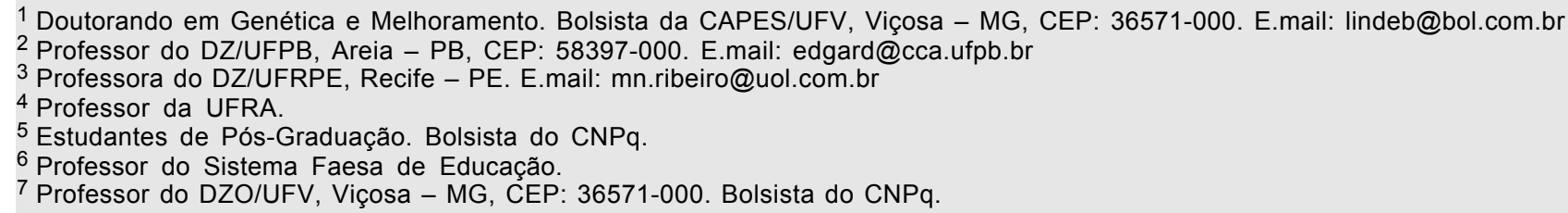


terística diminui o número de cabritos nascidos e aumenta o intervalo de gerações. Devido à sua importância, deve-se procurar identificar as causas que interferem sobre a característica, sejam as de ordem genética ou ambientais, mantendo o objetivo de elevar ao máximo o número de crias durante à vida reprodutiva da fêmea.

O intervalo de partos deve ser analisado em função dos objetivos da criação. Os sistemas de produção de carne e pele teriam maior rentabilidade com intervalos médios de 8 meses, ao passo que, em sistemas de produção de carne e leite, intervalos de aproximadamente 10 meses seriam desejáveis, pois possibilitariam períodos lactantes de 8 meses e mais 50 ou 60 dias de período seco. Entretanto, se o propósito for, fundamentalmente, leite, registra-se que a cabra tenha apenas uma parição por ano, com lactações médias de 10 meses (Simplício,1992).

Alguns fatores ambientais podem influenciar o intervalo de partos, dentre os quais estão o ano e a estação de ocorrência dos partos, o tipo de parto anterior ao intervalo, a idade ou ordem de parto e a duração da lactação.

Medeiros et al. (1982) estudaram a raça Bhuj no Piauí e observaram um intervalo médio de 246,20 \pm 53,6 dias com uma variação de 183 a 323 dias, enfatizando que o fator mais importante para essa variação está relacionado com a disponibilidade de alimentos por ocasião do parto em função da época do ano. Trabalhos com raças exóticas especializadas para produção de leite no Brasil, registraram intervalos de partos médios que variaram de 328,49 a 360,45 dias, dentre eles, pode-se citar Ribeiro (1997), Gonçalves et al. (1997) e Soares Filho et al. (2001).

Partos múltiplos podem ocasionar alterações no intervalo de partos posterior, estendendo-o devido ao estresse da cabra, uma vez que para gerar e manter a gestação de dois ou mais cabritos há uma maior mobilização de nutrientes para a gestação e lactação, fazendo com que seja necessário um maior período de recuperação da mãe até a próxima concepção (Galina et al., 1995).

Os períodos curtos de lactação refletem uma menor quantidade de leite produzido, já os longos estão associados à baixa eficiência reprodutiva do rebanho. Influências da duração da lactação sobre o primeiro intervalo de partos foram relatadas por Singh et al. (1970), e a regressão do intervalo de partos, em função da duração da lactação revelou aumento de 0,4 dias no intervalo de partos por dia de lactação.
A literatura nacional ainda é pobre no que diz respeito à estimativa de parâmetros genéticos para características reprodutivas em caprinos. De maneira geral, características reprodutivas em fêmeas possuem baixos coeficientes de herdabilidade e repetibilidade.

A estimativa de herdabilidade é essencial na determinação do método a ser utilizado em programas de melhoramento e seleção. Estimativas de herdabilidade para intervalo de partos, verificadas na literatura, apresentam valores variando de 0,02 a 0,16 (Ribeiro, 1997; Gonçalves et al., 1997; e Soares Filho et al., 2001).

Características que se repetem ao longo da vida do animal se expressam em diferentes intensidades em cada ocorrência. Valores de repetibilidade para intervalo de partos foram relatados por Ribeiro (1997), 0,09, e por Gonçalves et al. (1997), 0,10.

Objetiva-se com este trabalho avaliar a influência de efeitos de ambiente e estimar parâmetros genéticos para a característica de intervalo de partos de caprinos leiteiros criados no semi-árido paraibano, utilizando-se a metodologia da máxima verossimilhança restrita sob modelo animal.

\section{Material e Métodos}

Nesse estudo foram utilizados 486 registros de intervalo de partos de cabras mestiças paridas entre 1988 e 1996, oriundas do cruzamento de bodes pardo Alpino com cabras do tipo nativo Gurguéia, da Fazenda Carnaúba, pertencente à AMDA (Agropecuária Manoel Dantas Ltda), situada no município de Taperoá, microrregião do Cariri Ocidental da Paraíba.

A região se caracteriza por um clima seco, apresentando precipitações pluviométricas irregulares, girando em torno de $400 \mathrm{~mm}$ anuais de normalidade climática, ocorrendo maior concentração das chuvas nos meses de fevereiro a maio.

O sistema de criação é o semi-extensivo. $\mathrm{Na}$ época das chuvas, os animais eram soltos durante o dia para pastarem, retornando aos apriscos ao final do dia, onde permaneciam à noite. Recebiam concentrado protéico e energético no momento em que eram ordenhados, além de uma mistura mineral fornecida à vontade durante todo ano. Na época da seca, os animais permaneciam parte do dia à pasto e a outra, nas instalações, onde recebiam um complemento volumoso no cocho, à base de feno de capim-buffel (Cenchrus ciliares L.) e leguminosas nativas da 
região, palma forrageira picada (Opuntia ficus), capim-elefante picado (Penisetum purpureum) e raspa de mandioca (Manihot utilissima, Pohl).

Durante todo ano, as cabras permaneceram parte do dia no campo, sem a presença de rufião, sendo a deteç̧ão do cio feita visualmente pela manhã e à tarde. Caso fosse identificada, a cabra era levada à baia do reprodutor correspondente, onde ocorria a cobrição, e depois retornava ao lote. A monta era do tipo natural controlada, sem estação de monta.

Os cabritos recém-nascidos mamavam o colostro e eram retirados do pé da cabra, passando a receber leite de vaca em sistema de aleitamento artificial. Permaneciam no cabriteiro todos juntos até a ocasião do desmame aos 90 dias de idade, aproximadamente. Aos 120-150 dias de idade, as cabritas passavam para o lote das novilhas, onde eram cobertas na ocasião do primeiro cio fértil. Após a primeira parição, eram incorporadas no lote das cabras adultas.

Os dados foram obtidos de fichas individuais de matrizes caprinas paridas entre 1988 e 1998 controladas a cada 28 dias, que continham informações de genealogia, produção e reprodução.

Algumas restrições foram feitas nos dados, consideraram-se para análise animais nascidos entre 1988 e 1996; animais com efeitos fixos presentes (grupo contemporâneo, tipo de parto e ordem de parto); animais com pai e mãe conhecidos. Após as análises descritivas, foram mantidos bodes com, no mínimo, três filhas no rebanho, grupos contemporâneos com pelo menos dois animais, cabras com no máximo 12 parições e com intervalo de partos maiores que 170 e menores que 550 dias. Feitas as restrições restaram para análise 486 registros de intervalos de partos de 114 cabras, filhas de 17 bodes.

As análises dos efeitos ambientais foram realizadas pelo procedimento GLM dos $\operatorname{SAS}^{\circledR}$ (1999), considerando-se a opção RANDOM que trata o efeito de reprodutor como aleatório, utilizando o modelo estatístico abaixo:

$$
\begin{aligned}
y_{i j k l m}= & \mu+R_{i}+M_{j} / R_{i}+G C_{k}+T_{l}+b_{1}\left(I_{i j k l m}-\bar{I}\right)+ \\
& b_{2}\left(I_{i j k l m}-\bar{I}\right)^{2}+b_{3}\left(D L_{i j k l m}-\overline{D L}\right)+\varepsilon_{i j k l m}
\end{aligned}
$$

em que $Y_{\mathrm{ijklm}}$ é intervalo de partos do animal $m$ filha do bode i, da cabra $\mathrm{j}$ dentro do bode i, pertencente ao grupo contemporâneo k e com tipo de parição $1 ; \mu$, média geral; $R_{i}$, efeito aleatório do bode $i ; M_{j} / R_{i}$, efeito aninhado da cabra $\mathrm{j}$ com o bode $\mathrm{i} ; \mathrm{GC}_{\mathrm{k}}$, efeito fixo do grupo contemporâneo $\mathrm{k} ; \mathrm{T}_{1}$, efeito fixo de tipo de parto, sendo 1 correspondente ao parto simples e 2 ao múltiplo; $\mathrm{I}_{\mathrm{j} j \mathrm{klm}}$, efeito da idade da cabra ao parto, como covariável; $\bar{I}$, média da covariável; $\mathrm{b}_{1}$, coeficiente de regressão linear para idade da cabra ao parto; $\mathrm{b}_{2}$, coeficiente de regressão quadrático para idade da cabra ao parto; $\mathrm{DL}_{\mathrm{ijklm}}$, efeito da duração da lactação, como covariável; $b_{3}$, coeficiente de regressão linear para duração da lactação; $\overline{\mathrm{DL}}$, média da covariável; $\varepsilon_{\mathrm{ijklm}}$, erro aleatório independentemente distribuído com média zero e variância $\sigma^{2}$.

Os grupos contemporâneos foram formados pelo ano de parição $(1988, \ldots ., 1996)$ e a estação de parição (1 e 2), onde a estação 1 corresponde ao período chuvoso, que vai de fevereiro a junho, e a estação 2 , ao período seco, que vai julho a janeiro do ano seguinte.

As estimativas dos componentes de variância e parâmetros genéticos foram obtidos utilizando-se o aplicativo MTDFREML, descrito por BOLDMAN et al. (1995), que utiliza a metodologia da máxima verossimilhança restrita livre de derivadas.

O critério de convergência utilizado foi a variância dos valores do simplex inferior a $10^{-9}$. Após a convergência o programa era reiniciado usando as estimativas obtidas anteriormente como valores iniciais. Este procedimento foi repetido até que a diferença entre as estimativas das duas últimas convergências não diferissem. Os valores iniciais foram os obtidos com base na análise de variância efetuada pelo método dos quadrados mínimos.

A análise foi conduzida sob modelo animal, utilizando-se o seguinte modelo:

$$
Y=X b+Z_{1} d+Z_{2} p+\varepsilon
$$

em que $Y$ é vetor das observações n x $1 ; X$, matriz de incidência dos efeitos fixos $\mathrm{n} \times \mathrm{f} ; b$, vetor dos efeitos fixos $\mathrm{f} \times 1 ; Z_{1}$, matriz de incidência dos efeitos genéticos aditivos diretos $\mathrm{n} \times \mathrm{N} ; d$, vetor dos efeitos genéticos aditivos diretos $\mathrm{Nx} 1 ; Z_{2}$, matriz de incidência dos efeitos permanentes de meio $\mathrm{n} \times \mathrm{N} ; p$, vetor dos efeitos permanentes de meio $\mathrm{Nx} 1$; $\varepsilon$, vetor dos efeitos residuais $\mathrm{n} \times 1 \sim \mathrm{N}\left(0, \mathrm{I} \sigma_{\mathrm{e}}{ }^{2}\right)$, em que $\mathrm{n}=$ número de observações de intervalo de partos; $\mathrm{f}=$ número de níveis de efeitos fixos; $\mathrm{N}=$ número de animais com observação.

Os efeitos fixos considerados neste modelo foram os mesmos utilizados no modelo anterior, da análise dos efeitos ambientais. 


\section{Resultados e Discussão}

A média estimada para o intervalo de partos foi de $275,55 \pm 60,96$ dias com um CV de $22,12 \%$. Como o propósito do sistema de criação é leite e carne, o valor encontrado para esse rebanho está próximo ao relatado por Simplício (1992) como ideal. Essa média está acima da verificada por Medeiros et al. (1982) para a raça Bhuj no Nordeste brasileiro, porém inferior à relatada por Ribeiro (1997), em cabras Saanen no Sudeste, Gonçalves et al. (1997) para as raças Parda Alpina, Saanen e Toggenburg no Sudeste do Brasil e Soares Filho et al. (2001) para as raças Parda Alpina, Saanen e Toggenburg no Distrito Federal. Tal estimativa indica ainda eficiência em termos reprodutivos e evidencia a perfeita adaptação desses animais à região.

Todavia, melhores intervalos de partos poderiam ser alcançados através da redução do período de serviço, de modo a atingir a eficiência reprodutiva ótima, ou seja, três partos a cada dois anos.

A análise de variância revelou que os efeitos bode, cabra dentro de bode, grupo contemporâneo e duração da lactação mostraram-se significativos

\begin{tabular}{|c|c|c|c|}
\hline \multirow{2}{*}{$\begin{array}{l}\text { Fontes de variação } \\
\text { Source of variation }\end{array}$} & \multicolumn{2}{|c|}{ GL Quadrado médio } & \multirow{2}{*}{$\begin{array}{l}\mathrm{P}-\text { Valor } \\
P-\text { Value }\end{array}$} \\
\hline & $D F$ & Mean square & \\
\hline $\begin{array}{l}\text { Bode } \\
\text { Buck }\end{array}$ & 16 & 8364,53 & 0,0043 \\
\hline $\begin{array}{l}\text { Cabra dentro de bode } \\
\text { Goat inside of buck }\end{array}$ & 138 & 5529,33 & 0,0026 \\
\hline $\begin{array}{l}\text { Grupo contemporâneo } \\
\text { Contemporary group }\end{array}$ & 18 & 7656,41 & 0,0073 \\
\hline $\begin{array}{l}\text { Tipo de parto } \\
\text { Kidding type } \\
\text { Idade da cabra ao parto } \\
\text { Age of goat at kidding }\end{array}$ & 1 & 2944,09 & 0,3742 \\
\hline $\begin{array}{l}\text { Regressão linear } \\
\text { Linear regression }\end{array}$ & 1 & 3485,04 & 0,3337 \\
\hline $\begin{array}{l}\text { Regressão quadrática } \\
\text { Quadratic regression } \\
\text { Duração da lactação } \\
\text { Lactation length }\end{array}$ & 1 & 930,04 & 0,6172 \\
\hline $\begin{array}{l}\text { Regressão linear } \\
\text { Linear regression }\end{array}$ & 1 & 376906,76 & 0,0001 \\
\hline $\begin{array}{l}\text { Resíduo } \\
\text { Error }\end{array}$ & 297 & 3716,59 & \\
\hline
\end{tabular}

$(\mathrm{P}<0,05)$ sobre o intervalo de partos (Tabela 1$)$.

$\mathrm{O}$ efeito significativo do grupo contemporâneo sobre o intervalo de partos pode ser explicado pela variação do manejo proporcionada pela estacionalidade na produção de forragem. Resultado similar foi relatado por Medeiros et al. (1992), que atribuiu tal variação às diferenças de disponibilidade de forragem em termos qualitativos e quantitativos. Dessa forma, alguns ajuste no manejo alimentar poderiam amenizar as influências desse efeito.

$\mathrm{O}$ fato de o tipo de parto não influenciar o intervalo de partos deve-se ao manejo dos cabritos utilizados na fazenda, que são aleitados artificialmente, evidenciando a falta de estímulo diferenciado. A cabra ao receber estímulo de um cabrito é diferente do estímulo de dois ou três, onde, teoricamente, aumentaria a demanda de nutrientes por parte da gestação e para a síntese do leite, de modo a garantir o nascimento e sobrevivência das crias. Com isto, haveria aumento do período de serviço e, conseqüentemente, do intervalo de partos, pelo fato de a cabra necessitar de maior período de tempo para se recuperar e retornar à atividade reprodutiva. Como as cabras passam a receber estímulo único depois da parição, o do ordenhador, esse efeito seria anulado. Resultados similares foram obtidos por Ribeiro (1997) e Soares Filho (2001), porém o contrário foi obtido por Galina et al. (1995).

A não significância da idade da cabra ao parto pode ser explicada pela tendência de as fêmeas terem partos simples nas primeiras parições, ao contrário das fêmeas maduras, que tendem a ter partos múltiplos. Pode haver, então, equivalência da demanda de nutrientes para o crescimento e a manutenção da gestação, nas primíparas, pela maior demanda de nutrientes da barrigada múltipla, nas adultas. Resultados similares a este foram obtidos por Gonçalves et al. (1997) para cabras especializadas em produção de leite, no Sudeste do Brasil.

A duração da lactação exerceu influência sobre o intervalo de partos, sendo responsável por $26,56 \%$ da variação total para essa característica. A regressão do intervalo de partos, em função do período de lactação (Figura 1), revelou aumento de 0,59 dias no intervalo de partos para cada dia no período de lactação, corroborando o resultado obtido por Singh et al. (1970).

A estimativa de herdabilidade para o intervalo de partos foi de $0,09 \pm 0,069$, que é considerada de baixa magnitude, indicando que a característica é altamente 


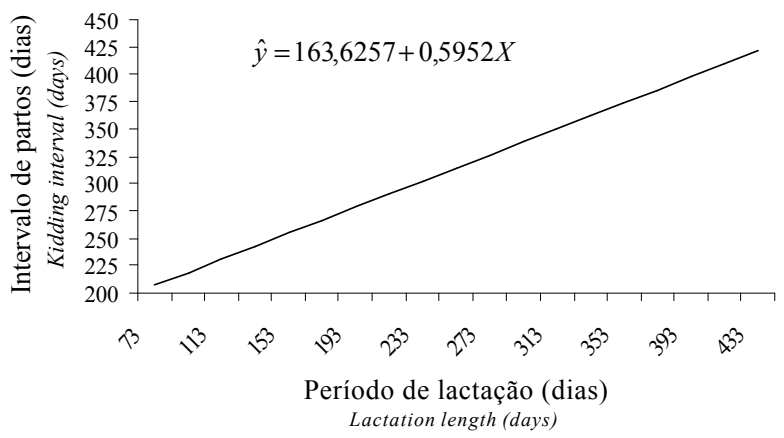

Figura 1 - Regressão do intervalo de partos, em função da duração da lactação.

Figure 1 - Regression of the kidding interval, according to the lactation length.

influenciada pelo ambiente. Esse valor encontra-se próximos dos verificados na literatura nacional. Resultado inferior foi obtido por Gonçalves et al. (1997), onde verificou uma herdabilidade de 0,046. Ribeiro (1997) e Soares Filho et al. (2001) obtiveram resultados semelhantes, com estimativas de herdabilidade de 0,09 e 0,10 , respectivamente.

A estimativa de repetibilidade do intervalo de partos foi de 0,09 $\pm 0,074$, devido ao fato de o componente de variância do ambiente permanente ser próximo a zero, fazendo com que a herdabilidade e a repetibilidade apresentassem praticamente os mesmos valores. Resultados semelhantes a esse foram obtidos por Ribeiro (1997) e Gonçalves et al. (1997), que estimaram a repetibilidade para o intervalo de partos de 0,09 e 0,10, respectivamente.

Dessa forma, pequeno progresso genético poderia ser alcançado caso esta característica fosse incluída em um programa de seleção, e respostas mais rápidas podem ser obtidas por melhoria nas práticas de manejo, como detecção de cio, interrupção da lactação e programas de alimentação bem definidos.

\section{Conclusões}

O intervalo de partos apresentou baixa herdabilidade e repetibilidade, o que levaria a pequenos ganhos genéticos por intermédio da seleção. Assim, reduções mais rápidas nessa característica podem ser obtidas pelo aprimoramento das práticas de manejo e condições de criação.

\section{Literatura Citada}

BOLDMAN, K.G.; KRIESE, L.A.; VAN VLECK, D.L. et al. A manual for use of MTDFREML A set of programs to obtain estimates of variances and covariances [DRAFT]. Lincoln: USDA/ARS, 1995. 120p.

GALINA, M.A.; SILVA, E.; MORALES, R. et al. Reproductive performance of mexican dairy goats under various management systems. Small Ruminants Research, v.18, p.249253, 1995.

GONÇALVES, H.C.; SILVA, M.A.; RAMOS, A.A. et al. Fatores genéticos e de meio no intervalo de partos de caprinos leiteiros. Revista Brasileira de Zootecnia, v.26, n.5, p.905-913, 1997.

MEDEIROS, L.P.; GIRÃO, R.N.; GIRÃO, E.S. et al. Produtividade de caprinos da raça Bhuj. Pesquisa Agropecuária Brasileira, v.17, n.9, p.1371-1375, 1982.

RIBEIRO, A.C. Estudo dos efeitos genéticos e de ambiente sobre características de importância econômica em caprinos da raça Saanen. Jaboticabal: Universidade Estadual Paulista, 1997. 85p. Dissertação (Mestrado) - Universidade Estadual Paulista, 1997.

STATISTICAL ANALYSIS SYSTEM. User's guide: Statistics. Version 8.0, Cary: 1999.

SIMPLÍCIO, A.A. Manejo reprodutivo e instalações. In: Curso de caprinocultura, 1992. Brasília, DF: ABEAS. n.5, pt 2, 53p.

SINGH, R.N.; ACHARYA, R.M.; BISWAS, D.K. Evolution of genetic and non-genetic factors affecting some economic traits in goats. Acta Agriculturae Scandinavica, v.20, p.10-14, 1970.

SOARES FILHO, G.; MCMANUS, C.; MARIANTE, A.S. Fatores genéticos e ambientais que influenciam algumas características de reprodução e produção de leite em cabras no Distrito Federal. Revista Brasileira de Zootecnia, v.30, n.1, p.53-59, 2001. 